Computing and Informatics, Vol. 39, 2020, 5 27 doi: 10.31577/cai_2020_1-2 5

\title{
FOG AND CLOUD COMPUTING ASSISTED IOT MODEL BASED PERSONAL EMERGENCY MONITORING AND DISEASES PREDICTION SERVICES
}

\author{
Zhancui Li
}

Department of Magnetic Resonance Surgery

The 960 Hospital of Joint Logistics Support Force of PLA

Shandong Taian, China

e-mail: lizc1027@163.com

Longri WeN*, Jimin LiU, Quanqiu JiA

Department of Information Engineering

Shandong University of Science and Technology

Shandong Taian, China

e-mail: wenlr51@sdust.edu.cn, jiaqq1995@163.com, skdljm@126.com

\section{Chengri CHE}

Department of Medical College Thoracic Surgery

Affiliated Hospital of Yanbian University

Jilin Yanji, China

e-mail: ycrche@ybu.edu.cn

\section{Chengfeng SHI, Haiying CAI}

Department of Maternal Healthcare, Maternal and Child Health Hospital Department of Cancer Prevention and Treatment Institute

Shandong Taian, China

e-mail: chengfengshi1980@163.com, haiyingcai1981@163.com

\footnotetext{
* Corresponding author
} 


\begin{abstract}
Along with the rapid development of modern high-tech and the change of people's awareness of healthy life, the demand for personal healthcare services is gradually increasing. The rapid progress of information and communication technology and medical and bio technology not only improves personal healthcare services, but also brings the fact that the human being has entered the era of longevity. At present, there are many researches focused on various wearable sensing devices and implant devices and Internet of Things in order to capture personal daily life health information more conveniently and effectively, and significant results have been obtained, such as fog computing. To provide personal healthcare services, the fog and cloud computing is an effective solution for sharing health information. The health big data analysis model can provide personal health situation reports on a daily basis, and the gene sequencing can provide hereditary disease prediction. However, the injury mortality and emergency diseases since long ago caused death and great pain for the family. And there are no effective rescue methods to save precious lives and no methods to predict the disease morbidity likelihood. The purpose of this research is to capture personal daily health information based on sensors and monitoring emergency situations with the help of fog computing and mobile applications, and disease prediction based on cloud computing and big data analysis. Through the comparison of test results it was proved that the proposed emergency monitoring based on fog and cloud computing and the diseases prediction model based on big data analysis not only gain more of the rescue time than the traditional emergency treatment method, but they also accumulate lots of different personal healthcare related experience. The Taian 960 hospital of PLA and the Yanbian Hospital as IM testbed were joined to provide emergency monitoring tests, and to ensure the CVD and CVA morbidity likelihood medical big data analysis, the people around Taian city participated in personal health tests. Through the project, the five network layers architecture and integrated MAPE-K Model based EMDPS platform not only made the cooperation between hospitals feasible to deal with emergency situations, but also the Internet medicine for the disease prediction was built.
\end{abstract}

Keywords: EMDPS, DML, PHR, EHR, IoT, fog computing, cloud computing, APC model

\title{
1 INTRODUCTION
}

Along with the rapid development of high technology and the improvement of personal healthcare, human beings have entered the era of longevity. However, the sudden death brings great pain to the family. From the age cohort analysis, the order of death causes is different. The top three causes of death in the children (1-14 years old) cohort were IM (injury mortalities), CA (cancer) and congenital abnormalities, accounting for $74.28 \%$, in the young adults cohort (15-44 years old) were IM, CA and CVD (cardiovascular disease), accounting for $75.97 \%$, in the middle-adult and aged (over 45 years old) cohort were CVD, CA and CVA 
(cerebral vascular accident), accounting for $88.07 \%$ [1]. Among them, most of CA and congenital anomaly belong to genetic disease, so the proposed EMDPS (emergency monitoring and diseases prediction service) model focused on the IM, CVD and CVA emergency situations monitoring and diseases morbidity likelihood analysis.

At present, despite the continuous development of medical technology and better rescue services, hospitals cannot accurately predict the DML (disease morbidity likelihood) and there is no advanced corresponding method for sudden death. In order to provide emergency forecasting and advanced rescue services the EMDPS model was proposed, and it is composed of the following modules.

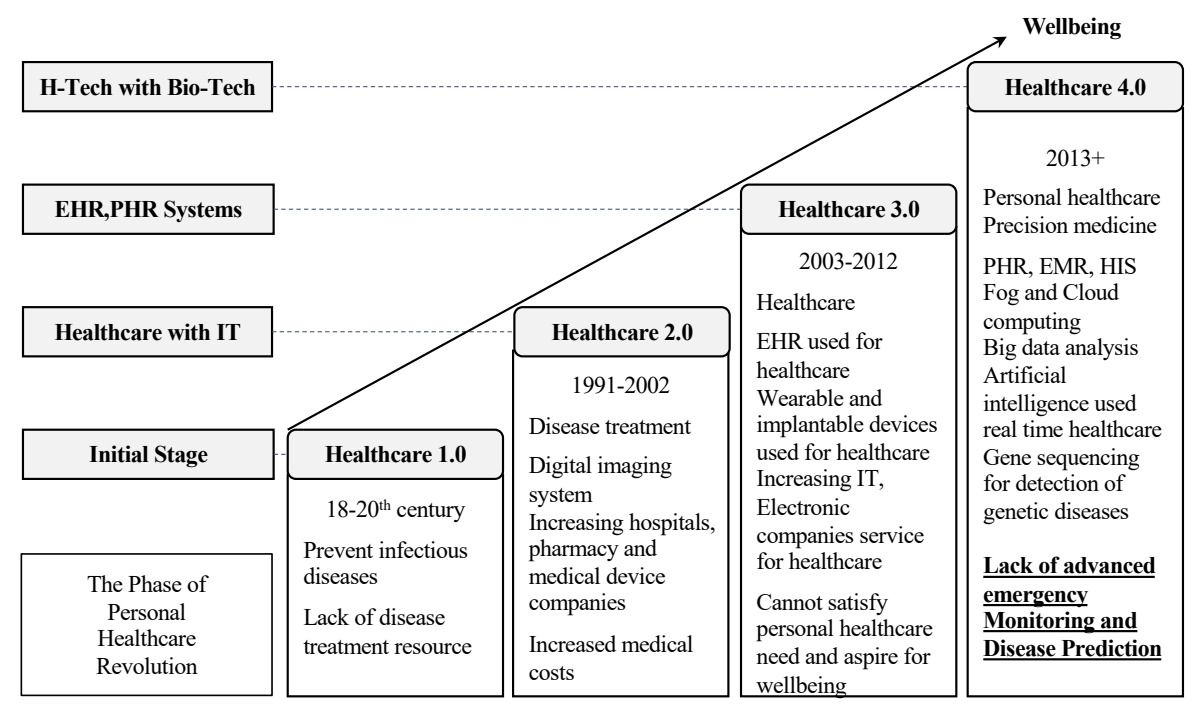

Figure 1. Revolution trend in China healthcare industry

The first is health information sensing module, the EMDPS model using PHR (personal health record) formation to capture information and screen emergency situation. The concept of PHR began to be used in 1978. PHR is personal health record generated in one's lifetime, it includes health-related information such as life log data, diagnostic record and genetic information and so on. Medical institutions use EMR (electronic medical record) and EHR (electronic health record) terms similar to PHR. EMR is a record used by a medical institution generated from medical information.

EHR is a generalized concept shared by multiple medical institutions [2]. The healthcare industry is developing rapidly, the healthcare technical level was upgraded from hospital-centered Healthcare 3.0 to current Healthcare 4.0 [3]. The difference between them is shown in Figure 1. At Healthcare 3.0, patients need to visit many hospitals and wait for a long time. Under the environment of Healthcare 4.0, the 
mechanism of EHR, PHR and fog and cloud computing based real-time data capture and delivery method can solve the problems of Healthcare 3.0.

The second is fog computing assisted IoT (Internet of Things) based personal daily life health information capture and monitoring module. Recently, the main services using PHR have been extended to diagnostic records, exercise information, and gene sequencing based application fields. Along with the development of IoT, linking multiple healthcare devices can collect a variety of personal health information, and the integrated applications should provide more services for personal healthcare 22. Fog computing has emerged as an active medical service solution because it contributes to the continuous monitoring of the health of remote patients and the detection of emergency situations. In addition, fog computing can reduce latency and communication costs, which is usually a huge problem in cloud computing. Fog computing is used to analyze, classify and share medical information between users and medical service providers [3]. The IoT based location position management model that makes use of the captured data resources could ensure the patient's personal information security and simplify the management. Even in emergencies, an efficient IoT healthcare service model can quickly respond using patient location information, so that hospital staff can locate patients in real time [4]. Subnet generation scheme, which collects and processes healthcare information to servers, provides a large amount of healthcare information through IoT devices connected by users. By assigning attribute values to the healthcare information sent to the server, a subnetwork is constructed according to the attribute values, and the related information between the subnetworks is extracted as seeds, and grouped into hierarchical structures. The server utilizes the deep operation of grouped medical information to extract optimized information and improve the observation speed and accuracy of decision making [5]. For the future Point-of-Care detection model the different sensing technologies were analyzed in detail, and that provided a path for the design and development of healthcare point detection device and data acquisition and processing in the future [6]. For the health information safe storage and accurate analysis, it is needed to transmit a large amount of captured data to the health cloud platform.

The third is cloud based health information delivery and secure store module. The personal healthcare cloud platform is a platform that can browse one's own health records ubiquitously and input health information and management independently. It is also a platform that can safely store and manage personal and family health information in one's lifetime. This platform not only provides a reliable technical basis for the capture and utilization of personal health information, but also provides a reliable experimental environment for the realization of precision medicine. A novel architecture for mobile group and cloud computing for healthcare could reduce costs, improve efficiency and reduce errors. At the same time, it could provide better consumer care and services for patients in the field of healthcare information to make them have universal transparency [7]. Cloud-MHMS (Cloud-based M-Health Monitoring System) puts the forward framework, which is used to achieve universal health information monitoring [8]. In 
terms of storage management, data analysis and data security management of health information, there are advanced management models and algorithms proposed [9, 10].

The fourth is health big data analysis based emergency monitoring and disease prediction module. Personal healthcare platform as a new medical service technology or application tool not only improves the accuracy of diagnosis and disease prediction, but it is also improving the quality of life. Many data analysis methods have been applied in the field of disease prediction [11, 12]. Among them, the APC (Age Period Cohort) model and ANN (Artificial Neural Network) based disease prediction has made remarkable achievements. The APC model based on age, period and cohort analysis needs fewer original data attributes in disease prediction, but it better reflects the trend of health status than other models. The proposed APC model establishes a prospective cohort model by analyzing the three impact factors of test result for the DML prediction.

The last one is health situation visualization and rescue service module. It is the purpose of the research that captures personal health information in real time and provides healthcare services according to a personal health situation. The MPR (medication possession ratio) monitoring application is a supervisor medication related decision making method to enhance the analysis function of personal health records [13, 14, and it is a good reference for our research. In order to provide a personal healthcare more quickly, effectively and accurately, a personal health situation visualization EMS (Emergency Monitoring Services) and DPS (Disease Prediction Services) applications were proposed.

\section{THE EMDPS NETWORK ARCHITECTURE}

The fog and cloud computing assisted IoT architecture is a network scenario where everything is connected and uniquely identified over the global information and communication infrastructure [15]. The traditional IoT architectures can be decomposed into three layers, as shown in Figure 2 .

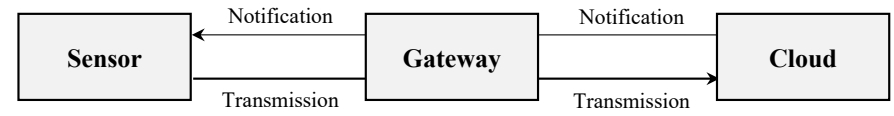

Figure 2. IoT-based system for a remote patient monitoring

The first layer is sensor, it is used for health information capture and delivery. The second layer is gateway, for the health information monitoring, it only acts as a relay between sensor and cloud. Gateway provides continuous, conventional and safe communication services with sensors using different network protocols such as Wi-Fi, ZigBee, and Bluetooth. The third layer is cloud, a broad health big data analysis, it safely stores amount of personal daily health sensory data and accurately predicts diseases morbidity likelihood. The latency is critical impact factor of IoT 
network performance and the case core network was added in Cisco fog and IoT distributed architecture, as shown in Figure 3. The core network could provide paths to carry and transfer data and network information between numerous subnetworks and protect against network threats [16].

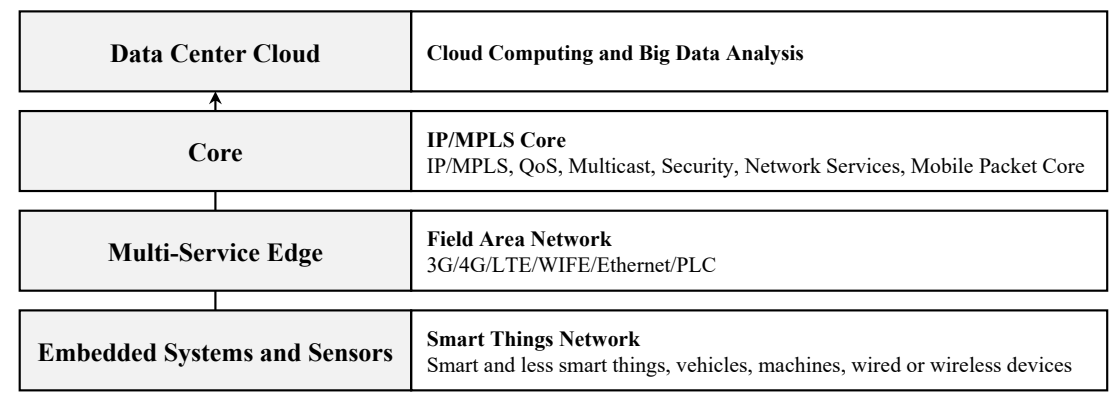

Figure 3. Cisco fog and IoT distributed architecture

Considering the impact factor of network latency in emergency situations and information feedback from the rescue service center, the mobile edge network layer is added to the proposed EMDP architecture, as shown in Figure 4.

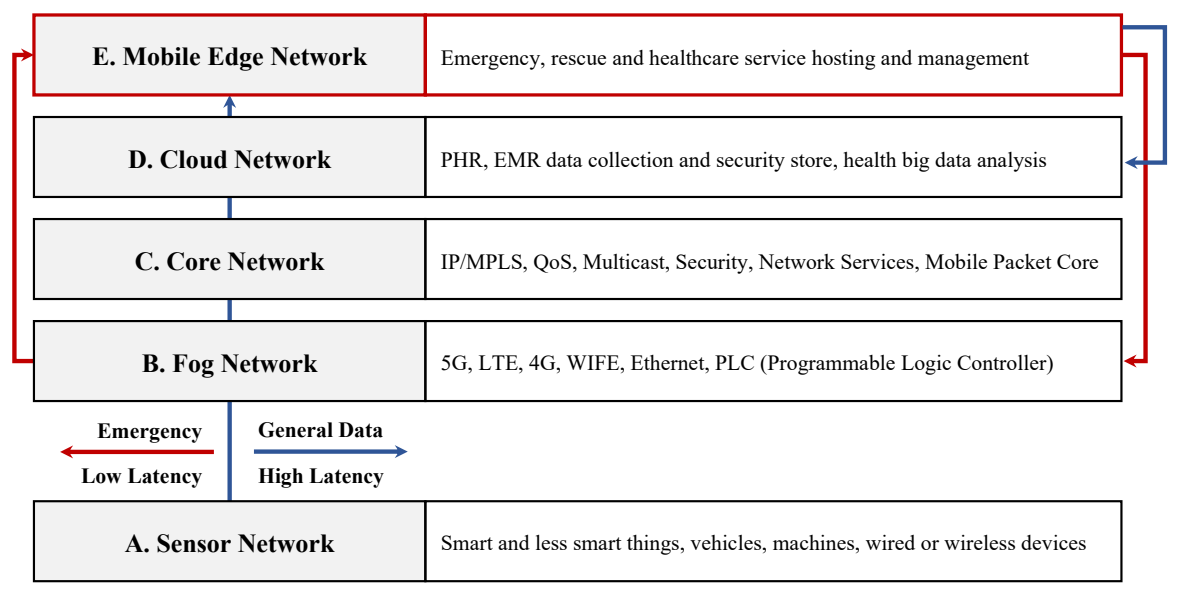

Figure 4. The proposed EMDPS network architecture

The EMDP architecture consists of four layers. The emergency ubiquitously monitoring finds a way in the fog network layer, the diseases morbidity likelihood is analyzed in the cloud network layer, and the mobile edge network layer is an actuator which provides the service feedback to the fog and cloud network layer. 


\subsection{Sensor Network Layer}

The sensor device focused on arrhythmia detection for the IM, CVD and CVA emergency situations monitoring in the EMDP model, and PHR related information from the personal daily health information was captured by additional devices such as smart watch and flexible and stretchable physical sensors. Recently, flexible and stretchable physical sensors such as temperature, pressure, and strain sensors that can measure and quantify electrical signals generated by human activities are attracting a great deal of attention as they have unique characteristics [17]. There are ECG (Electrocardiogram) [18], RESP (Respiration), and NIBP (Non-Invasive Blood Pressure), SPO2 (Surplus Pulse O2) could be detected using smart and less smart sensor devices [19.

\subsection{Fog Network Layer}

Using the fog network core character of low latency and location awareness, the very large number of fog nodes could receive emergency information in real time, wireless, and heterogeneous ways from the sensor network layer and send it to the mobile edge network layer [21, 22, 23. In this way, EMDPS model could save a lot of time to deal with emergency situations. For disease prediction, the fog layer sends periodic PHR to the cloud network layer using a standardized data format.

\subsection{Core Network Layer}

The core network is similar like in traditional networks and provides paths to carry and transfer data and network information between numerous subnetworks. The traffic profile is the critical variation between IoT and traditional core network layers [16]. The core network layer not only provides the best network from fog to cloud network layer but also provides QoS and data transmission security for the EMDPS model.

\subsection{Cloud Network Layer}

The main task of the cloud network is to receive the PHR from fog network and the EHR from the medical institutions, and store it safely in a standardized form and manage it. The cloud based PHR platform configuration was proposed in the previous research and offered the way how to provide healthcare services for aged cohort [23, 9, 10]. In order to ensure a more efficient transmission and network architecture the customized architecture [24, a novel architecture [7] and fine grained access architecture [25] for the cloud network were referenced for the EMDPS cloud network design. For the disease prediction, the APC (Age Period Cohort) model based health big data analysis services were provided in this layer. 


\subsection{Mobile Edge Network Layer}

Recently, many mobile applications for the personal healthcare have appeared on the market. The IoT application healthcare system [26], a distributed movement prediction scheme [27] and the mobile phone based blood glucose management system [28, 8] were researched for our mobile application services.

At the emergency situation, the mobile edge network layer analyses received monitoring results from the fog network layer and delivers it to the family members, neighborhood service center and special mobile vehicle service center and nearby hospitals. Whenever received any acknowledgement from these service centers, the mobile application sends a feedback to the user and fog network layer as soon as possible.

Normally, it will receive periodical PHR and EHR analysis result from the cloud and provide health visualization service for the users. Because of the high morbidity likelihood of diseases such as CVD and CVA, it will send the related analysis reports and notify on the health situation the user medical institutions and family members. When any request from the user healthcare supporter is received, it sends a feedback to the cloud platform for the deep analysis and it decides on the further healthcare service.

\section{THE EMDPS PLATFROM ARCHITECTURE}

The IBM's MAPE-K model is an alternative computing model that provides automated management components for computational units and specifies system behaviors. The MAPE-K (Monitor-Analysis-Plan-Execute plus Knowledge) model is specified on the level of four different computing components: Monitor, Analyze, Plan, and Execute with the access to a partially or fully shared knowledge base, as shown in Figure 5. The Monitor captures health information from sensors, and it is the closest to the sensor devices, also it can determine events attributes [29]. The Analyze selects the data formation and analysis model. The Plan is in charge of selecting or generating a procedure for the system. The Execute provides necessary changes in the system and determines the behavior of the system [30].

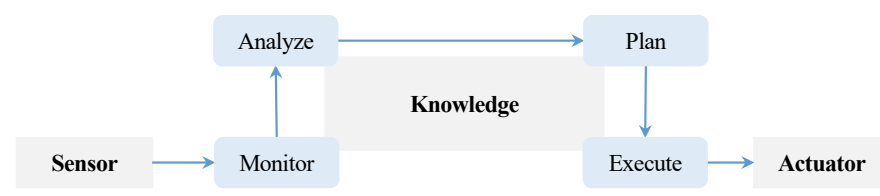

Figure 5. IBM's MAPE-K model

To fulfill the desired closed-loop behavior for resource management, the enhanced MAPE-K model was proposed in HICH (Hierarchical Fog-Assisted Computing Architecture for Healthcare IoT) [15], in which a new component System 
Management is integrated. The four MAPE-K components are enabled with feedback in the model. The feedback is received from Execute, System Management is used to periodically tune the computing components with respect to the inputs and the computations in the model, as shown in Figure 6.

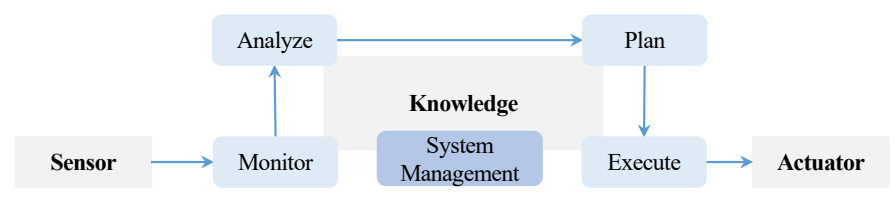

Figure 6. The HICH enhanced MAPE-K model

To efficiently deal with emergencies the mobile edge network layer was added to the EMDPS architecture. According to the characteristics of the proposed EMDPS architecture, the MAPE-K model was proposed, also based on the HICH model, integrating the Analyze separated by fog and cloud layer, and EMS and DPS as the Plan. As the result, it not only retains the MAPE-K model original function but also implements the HICH model system management function. Focused on the emergencies and network latency, the EMDPS model using distributed computing method replaced the original Plan components using EMS and DPS, as shown in Figure 7

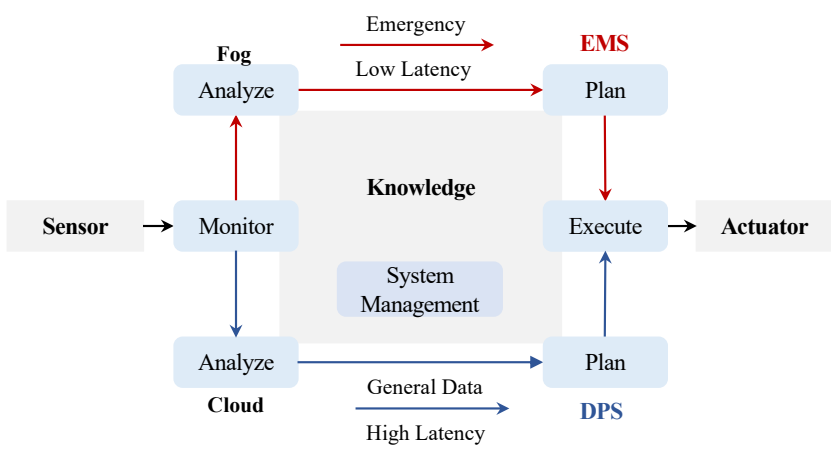

Figure 7. The EMDPS integrated MAPE-K model

\subsection{EMDPS Platform Architecture}

The proposed EMDPS platform is designed to provide a personal health care and related medical services. The service users are divided into three different groups. The main user group includes people and patients who want to receive their personal health care through the platform. The second user group is the family members and the personal health manager. The third user group represents medical institutions, 
healthcare centers and emergency response service providers. All the platform users are connected and communicate through the web site and mobile applications.

The main user group are people who use the personal smart devices to detect physical fitness and healthy ingredients, such as BPG (Blood Pressure Gauge), BGM (Blood Glucose Meter), and so on. And these personal health information will be sent to the gateway server of the residence community health care center. The EMDPS platform partitions the health data analytics into two parts: the emergency data analysis running on fog nodes and PHR based diseases prediction analysis in the cloud.

The EMDPS platform provides EMS and DPS on mobile edge node and the knowledge is distributed to different layers. The EMDPS deploys EMS and DPS closed System Management base of emergency situations. The preselection could be determined according to the emergency monitoring parameters. The EMDPS Platform can be used to different resource system management, although the focus is on the network traffic management to efficiently deal with a personal health emergency situation and accuracy prediction of diseases morbidity likelihood, as shown in Figure 8

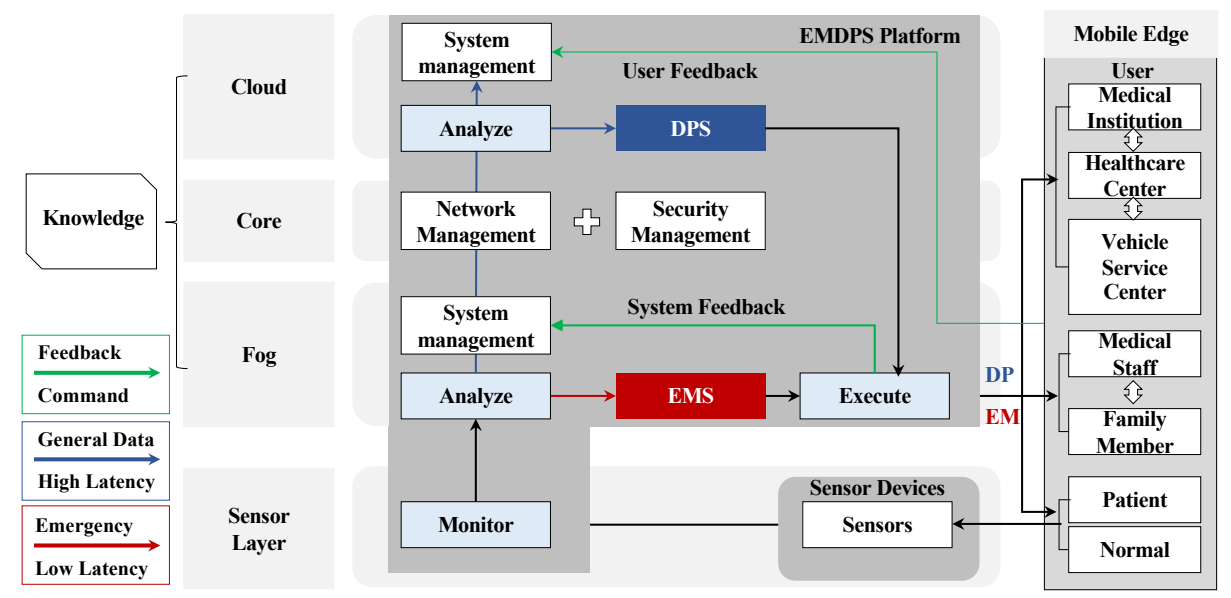

Figure 8. The proposed EMDPS platform configuration

In order to solve the problem of the network delay, the EMDPS platform uses the proposed five-layer network architecture. To deal with EM and DP situations correctly, the EMDPS platform uses integrated MAPE-K model.

\subsection{Emergency Monitoring Method}

The sensors could periodically capture personal health information and transmit the monitoring report to the gateway (fog layer), see the report format in Table 1 . There are two types of monitoring reports from the Monitor component, the one is 
emergency and the other is PHR. When it is an emergency, the emergency report with blood pressure, location and remarks will be sent to the fog node as soon as possible.

\begin{tabular}{|c|c|c|c|c|c|c|c|}
\hline Manual & \multicolumn{3}{|c|}{ Situation } & \multicolumn{2}{|c|}{ Systolic Pressure } & \multicolumn{2}{|c|}{ Remarks } \\
\hline \multirow{5}{*}{ Monitor } & \multicolumn{2}{|c|}{ Emergency } & PHR & High & Normal, Low & IM & Diseases \\
\hline & & & 0 & 1 & 0 & 1 & 0 \\
\hline & \multicolumn{3}{|c|}{ Diastolic Pressure } & \multicolumn{2}{|c|}{ Location } & \multicolumn{2}{|c|}{ Family } \\
\hline & Low & \multicolumn{2}{|c|}{ Normal, High } & System Located & Personal Located & Contact & None \\
\hline & 1 & \multicolumn{2}{|r|}{0} & 1 & 0 & 1 & 0 \\
\hline
\end{tabular}

Table 1. Emergency report on sensor layer monitor

As soon as the fog receives the emergency report from the sensor layer, it will analyze the personal information mapping to the captured report information and send EMS as a plan to the mobile edge layer for the user, the report format is presented in Table 2. There are several previous researches for the fog computing and health data analysis [31], for the goal of an efficient emergency monitoring report and accuracy service, which defined several critical factors for the rescue.

\begin{tabular}{|c|c|c|c|c|c|c|c|c|}
\hline Manual & Situation & \multicolumn{3}{|c|}{ Blood Pressure } & \multicolumn{3}{|c|}{ Location } \\
\hline \multirow{4}{*}{ Analyze } & Emergency & Systolic & Diastolic & None & \multicolumn{3}{|c|}{ Map Add } \\
\cline { 2 - 9 } & \multicolumn{2}{|c|}{1} & \multicolumn{2}{|c|}{ sy } & \multicolumn{2}{|c|}{ di } & null & \multicolumn{3}{|c|}{ ad } \\
& \multicolumn{2}{|c|}{ Age } & \multicolumn{3}{|c|}{ Gender } & \multicolumn{3}{c|}{ EMS } \\
\cline { 2 - 9 } & Age & None & Male & Female & Others & FM & HEC & VSC \\
\cline { 2 - 5 } & ag & Null & 1 & 0 & null & null & null & null \\
\hline
\end{tabular}

Table 2. Emergency report on fog layer analysis

In the emergency situation, EM method uses five impact factors for the rescue. The first is blood pressure, it will determine the user health situations of IM or disease. The second is gender, the third is age. The range of normal blood pressure varies according to sex and age factor and all the information could be helpful for rescue service. The fourth is user location information, it will be send to the service center in real time and used to contact rescue service staff and family member. The last one is EMS, it will contact FM (family member), HEC (hospital emergency center) and VS (vehicle service center) such as DIDI vehicle service center. The emergency monitoring report will also send system feedback to the cloud for the continuous rescue service and EMS mapping with platform PHR and EHR.

\subsection{Disease Prediction Method}

It is difficult to share personal EHR from the medical institutions, so the daily personal health information collected by EMDPS platform and medical reports col- 
lected by medical research institutes are used as data sources for disease prediction test. The DPS focus on CVD and CVD morbidity likelihood prediction in this research to prevent sudden death from the diseases. In order to better share personal health information and easy to exchange utilization, the standardized PHR model is proposed based on general PHR, hospital EHR and medical reports; see the report format in Table 3 .

\begin{tabular}{|c|c|c|c|c|c|c|}
\hline Manual & Situation & Gender & Age & \multicolumn{2}{|c|}{ Temperature } & Puls Rate \\
\hline Analyze & PHR & Null & Null & & Null & Null \\
\hline \multirow{4}{*}{ General } & 0 & & & \multirow{2}{*}{\multicolumn{2}{|c|}{ Blood Pressure }} & $($ Times/M) \\
\hline & Height & Weight & BMI & & & Respiratory Rate \\
\hline & Null & Null & Null & sy & $\begin{array}{l}\mathrm{di} \\
\end{array}$ & Null \\
\hline & $(\mathrm{cm})$ & $(\mathrm{kg})$ & $(\mathrm{Kg} / \mathrm{m} 2)$ & Null & Null & (Times/Min) \\
\hline \multirow{4}{*}{ Habits } & \multicolumn{4}{|c|}{ Physical Training } & \multicolumn{2}{|r|}{ Eating } \\
\hline & \multicolumn{3}{|c|}{$\begin{array}{l}\text { 1. Daily, } \\
\text { 2. More than Once a Week, } \\
\text { 3. Occasionally, } \\
\text { 4. No Exercise. }\end{array}$} & & \multicolumn{2}{|c|}{$\begin{array}{l}\text { 1. Balanced, } \\
\text { 2. Buckwheat, } \\
\text { 3. Vegetarian, } \\
\text { 4. Halophilic, } \\
\text { 5. Oil Loving, } \\
\text { 6. Sugar Tolerance. }\end{array}$} \\
\hline & \multicolumn{3}{|c|}{ Smoking } & & \multicolumn{2}{|c|}{ Drinking } \\
\hline & \multicolumn{4}{|l|}{$\begin{array}{l}\text { 1. Never, } \\
\text { 2. Quit, } \\
\text { 3. Smoking. }\end{array}$} & \multicolumn{2}{|c|}{$\begin{array}{l}\text { 1. Never, } \\
\text { 2. Occasionally, } \\
\text { 3. Often, } \\
\text { 4. Daily. }\end{array}$} \\
\hline \multirow{4}{*}{ Diseases } & \multicolumn{4}{|c|}{ CVD } & \multicolumn{2}{|c|}{ Heart Disease } \\
\hline & \multicolumn{4}{|c|}{$\begin{array}{l}\text { 1. None, } \\
\text { 2. Ischemic Stroke, } \\
\text { 3. Cerebral Hemorrhage, } \\
\text { 4. Subarachnoid Hemorrhage, } \\
\text { 5. Transient Ischemic Attack, } \\
\text { 6. Others. }\end{array}$} & \multicolumn{2}{|c|}{$\begin{array}{l}\text { 1. None, } \\
\text { 2. Myocardial Infarction, } \\
\text { 3. Angina Pectoris, } \\
\text { 4. Revascularization of Coronary Artery, } \\
\text { 5. Congestive Heart Failure, } \\
\text { 6. Anterior Cardiac Pain, 7. Others. }\end{array}$} \\
\hline & \multicolumn{4}{|c|}{ CVA } & \multicolumn{2}{|c|}{ Others } \\
\hline & \multicolumn{4}{|c|}{$\begin{array}{l}\text { 1. None, 2. Dissecting Aneurysms, } \\
\text { 3. Arterial Occlusive Diseases, 4. Others. }\end{array}$} & \\
\hline \multirow{4}{*}{$\begin{array}{l}\text { Cases } \\
\text { of Treatment }\end{array}$} & \multicolumn{6}{|c|}{ Personal Medical History } \\
\hline & \multirow{2}{*}{\multicolumn{6}{|c|}{ Family Medical History }} \\
\hline & & & & & & \\
\hline & Cause of & Null & & & & \\
\hline
\end{tabular}

Table 3. Disease prediction report on cloud analysis

The APC (Age Period Cohort) is a generalized model proposed in 1939. It has three impact factors: age, period and cohort. The age factor impacts the results with the personal physiological changes and the accumulation of social experiences or social status changes. The period has impact on the result considering the lifetime and living surrounding. The cohort changes result from the cross-impact of the personal experience and social layer.

However, the APC model is a generalized linear model. There is a complete linear relationship between age and period and cohort variables, the period equals sum of the age and cohort value. The model design matrix is a singular matrix with non-full rank. The matrix is irreversible, so the unique solution of model parameters cannot be obtained. Therefore, as there is an "unrecognizable problem", a large number of parameter estimates exist. The method has been proposed [32], 
in which IE (intrinsic estimator) proposed by $\mathrm{Fu}$ does not need prior information assumptions, and it is close to the results of traditional generalized linear model. The endogenous factor estimator is convergent and unique, which is suitable for APC model parameter estimation 33.

The APC model based on time series can achieve the goal of disease prediction through the coefficients of each cohort. The basic form of the APC model is Formula (1).

$$
\ln \left[E\left(r_{i j k}\right)\right]=\ln \left(\Theta_{i j k} / N_{i j k}\right)=v+\alpha_{i}+\theta_{j}+\gamma_{k}+\varepsilon_{i j k}
$$

where $E\left(r_{i j k}\right)$ is the expected value of disease morbidity likelihood for the age cohort $(i)$, the period $(j)$ and the birth cohort $(k)$, the $\Theta_{i j k}$ represents the expected value of disease morbidity likelihood for the $i$ age cohort observed in the $j$ period, and $N_{i j k}$ represents the population in the corresponding age, period and birth cohort. The $v$ is the intercept of the regression model, the $\alpha_{i}$ is the impact of the $i^{\text {th }}$ age cohort, the $\theta_{j}$ is the impact of the $j^{\text {th }}$ period, the $\gamma_{k}$ is the impact of the $k^{\text {th }}$ cohort and the $\varepsilon_{i j k}$ is the random error.

The proposed disease prediction uses the IE integrated APC model and the 7284 males' and 8593 females' physical examination reports as the test sample data. In the test result, the CVA and CVD were marked as "2", and normal as " 1 ". The whole test was carried out with STATA 15, and the final results are shown in Table 4.

In the Age cohort, both men and women have a tendency to increase the risk of disease with age, but women may suffer from CVA and CVD earlier than men, which is related to the fact that women are more likely to suffer from hypertension and other diseases. Overall, Age growth has a significant impact on the DML. In the Period cohort, we can see the DML downward trend, which means the personal healthcare service and the people's awareness of health is growing.

In the birth cohort, we see a more complex result. It means that the male's DML shows a growth trend, on the contrary, there is a downward trend regarding females. The results are closely related to the personal lifestyle, bad habits such as smoking and drinking can lead to diseases, females pay more attention to health. Despite of that a higher CVA and CVD morbidity likelihood was noticed. According to the PHR analysis, the CVA and CVD morbidity likelihood was obtained. The proposed APC model is more suitable for personal disease prediction, but even having the insufficient sample data will lead to prediction accuracy.

The traditional APC model can predict disease through the age, period and birth cohort. It is observed from the data set that the smoking and drinking is the influence factor causing CVA and CVD.

For the test, 500 male and 100 female data were selected from the source data. Each group of data consists of two different user data of the same age, period and birth, but one of them is smoking or drinking. Calculate the $\ln \left[E\left(r_{i j k}\right)\right]$ of each data according to Formula (1), and then take the $\ln \left[E\left(r_{i j k}\right)\right]$ difference of two different 


\begin{tabular}{|c|c|c|c|c|}
\hline \multirow{2}{*}{ Cohort } & \multicolumn{2}{|c|}{ Male } & \multicolumn{2}{|c|}{ Female } \\
\hline & Coef. & OIM Std.Err. & Coef. & OIM Std.Err. \\
\hline Age_40 & -0.03007 & 0.06761 & -0.04307 & 0.07887 \\
\hline Age_45 & -0.02556 & 0.05431 & -0.01834 & 0.05186 \\
\hline Age_50 & -0.04382 & 0.05478 & -0.04237 & 0.05198 \\
\hline Age_55 & -0.0494 & 0.04889 & -0.04465 & $0.05058^{*}$ \\
\hline Age_60 & -0.05107 & 0.03423 & 0.00053 & 0.03582 \\
\hline Age_65 & $-0.0458^{* *}$ & 0.02369 & 0.00703 & $0.0257^{*}$ \\
\hline Age_70 & $0.02934^{*}$ & 0.01697 & 0.015703 & 0.01706 \\
\hline Age_75 & $0.033^{* * *}$ & 0.0165 & 0.01408 & 0.01402 \\
\hline Age_80 & $0.04023^{*}$ & 0.02303 & 0.01339 & $0.019 * * *$ \\
\hline Age_85 & $0.091^{* * *}$ & 0.03575 & 0.02566 & 0.03144 \\
\hline Period_2005 & $0.028^{* * *}$ & 0.01247 & 0.01473 & 0.0127 \\
\hline Period_2010 & -0.00376 & 0.00604 & -0.00692 & 0.00538 \\
\hline Period_2015 & $-0.018^{* * *}$ & 0.01254 & $-0.015^{* * *}$ & 0.01234 \\
\hline Birth_1925 & -0.01836 & 0.04652 & 0.00896 & 0.0464 \\
\hline Birth_1930 & -0.03144 & 0.02871 & 0.02014 & 0.02917 \\
\hline Birth_1935 & -0.03102 & 0.0194 & 0.01277 & 0.02001 \\
\hline Birth_1940 & -0.0104 & 0.0177 & 0.0109 & 0.0181 \\
\hline Birth_1945 & 0.00385 & 0.02324 & 0.00641 & 0.02345 \\
\hline Birth_1950 & 0.03012 & 0.03204 & -0.0296 & 0.03218 \\
\hline Birth_1955 & 0.0289 & 0.41947 & $-0.048^{* * *}$ & 0.04226 \\
\hline Birth_1960 & 0.01233 & 0.05335 & -0.01994 & 0.05121 \\
\hline Birth_1965 & $0.123^{* * *}$ & 0.0585 & -0.00241 & 0.05694 \\
\hline Birth_1970 & $0.1084^{* *}$ & 0.05547 & $-0.066^{* * *}$ & 0.05755 \\
\hline Birth_1975 & $0.256^{* * *}$ & 0.10157 & 0.02922 & 0.0662 \\
\hline Birth_1980 & 0.22342 & 0.12878 & 0.07748 & 0.06619 \\
\hline \multicolumn{5}{|c|}{$\begin{array}{l}\left({ }^{* *} p<0.0001 ;{ }^{* *} p<0.001 ;{ }^{*} p<0.05 ; \text { OIM.std.err: }\right. \\
\text { The square root of Coef's variance. } \\
\text { The Coef's calculates based on the observed information matrix } \\
\text { in the maximum likelihood estimation.) }\end{array}$} \\
\hline
\end{tabular}

Table 4. Disease prediction report on cloud analysis

user data in each cohort, and adjust the effect of these factors on the results using the Formula (2).

$$
\varepsilon_{i j k}=\sum_{1}^{n}\left(\ln \left[E_{A}\left(r_{i j k}\right)\right]-\ln \left[E_{B}\left(r_{i j k}\right)\right]\right) / n
$$

where $\varepsilon_{i j k}$ is the impact value of smoking on the results, $\sum_{1}^{n}\left(\ln \left[E_{A}\left(r_{i j k}\right)\right]\right.$ $\left.-\ln \left[E_{B}\left(r_{i j k}\right)\right]\right)$ is the result of different habit of $n$ groups in the same period, age and cohort, and $n$ is the total number of groups. The impact value of drinking on the result can be calculated in the same way. 
The influence of single factor (smoking or drinking) and multiple factors (drinking and smoking) to the test result is not simply cumulative outcome. In this case, we selected 200 male and 100 female groups sample data for the test. The influence of smoking using Formula (3) and the influence of drinking using Formula (4) also the multiple factors using Formula (5). The influence of smoking and drinking on diseases is shown in Table 5 .

$$
\begin{gathered}
\ln _{\text {smoke }}=\ln \left(\theta_{i j k} / N_{i j k}\right)=v+\alpha_{i}+\theta_{j}+\gamma_{k}+\varepsilon_{\text {smoke }}, \\
\ln _{\text {drink }}=\ln \left(\theta_{i j k} / N_{i j k}\right)=v+\alpha_{i}+\theta_{j}+\gamma_{k}+\varepsilon_{\text {drink }}, \\
\ln _{s+d}=\ln \left(\theta_{i j k} / N_{i j k}\right)=v+\alpha_{i}+\theta_{j}+\gamma_{k}+\varepsilon_{s+d} .
\end{gathered}
$$

\begin{tabular}{|l|r|r|r|r|}
\hline \multirow{2}{*}{ Habit } & \multicolumn{2}{|c|}{ Male } & \multicolumn{2}{c|}{ Female } \\
& Coef. & OIM Std. Err. & Coef. & OIM Std. Err. \\
\hline Smoke & 0.10369 & 0.06192 & 0.12469 & 0.06197 \\
\hline Drink & 0.09635 & 0.07362 & 0.10394 & 0.10377 \\
\hline Smoke + Drink & 0.13681 & 0.09684 & 0.14332 & 0.08251 \\
\hline
\end{tabular}

Table 5. The influence of smoking and drinking on diseases

Different prediction models can be selected by classifying the user data, but the accuracy has not been improved as expected after adding the intercept influence factors. The less control data groups of the same period, age and cohort cause the inaccuracy of the result simulation. At the same time, the lack of the number of women smoking and drinking control groups and the disproportion with men lead to the inaccuracy of the result. Today, the number of women smoking and drinking control groups is not enough.

\section{EMPDS PERSONAL HEALTCARE SERVICES}

The implemented healthcare services based on proposed EMDPS model are shown in Figure 9

The EMDPS platform consists of two main services.

The one is the emergency monitoring service. In order to provide this service, the management service node has been setup in the campus big data center and hospital data center, and also the residential community center.

At first, wearable devices collect users' health information and the detected information will be automatically stored in the mobile by personal health care application. The application will intelligently judge the collected health information. Such as blood pressure, it will judge systolic blood pressure and diastolic blood pressure checking whether the measured values are in the normal range. If the blood pressure passes beyond the standard value reaching the hazard value then the application will trigger the emergency and sends the analyze request to the service node. 


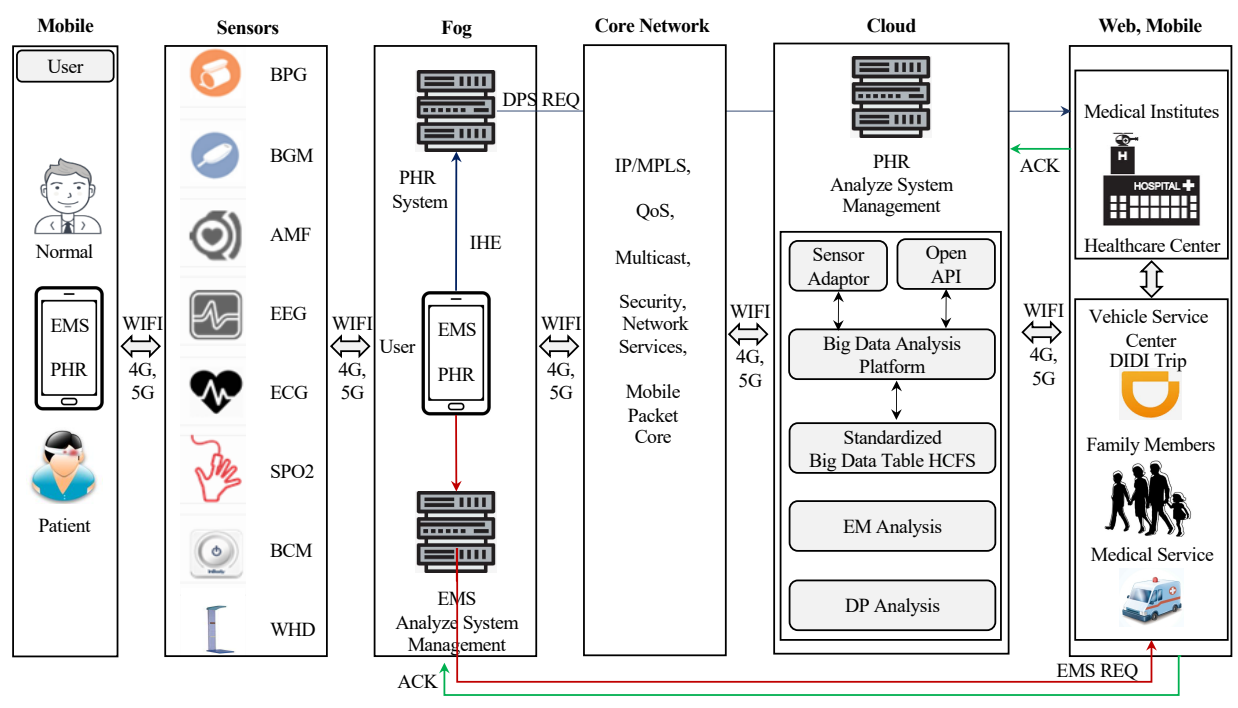

Figure 9. The proposed EMDPS healthcare service configuration

Secondly, when the fog service node receives EMS service it will send an EMS report to the medical institutes and a family member and also to a special vehicle service center.

At last, when the service center confirms the information, they will respond to the fog service node and request the stored user's personal health information and corresponding scheme from the cloud node.

The other one is the diseases prediction service. Because a large amount of a personal health information is collected every day and personal network conditions are different, it needs the support of cloud computing to safely store and analyze the daily health big data.

At first, the fog node analyzes the users' health information and if there is none emergency the collected data is sent to the cloud platform. Secondly, the collected personal daily health information will be modified to a standardized format and encrypted stored in the cloud storage. Finally, the results of APC based disease prediction model analysis will be sent to related medical institutions and to a personal user.

\section{TEST RESULT COMPARISON}

The EMS focuses on rescue time reduction, and the DPS is focused on diseases accurate prediction and effective personal healthcare services. So the test results are compared with the traditional services regarding the time of the rescue service and modified healthcare services. 


\subsection{Emergency Monitoring Test Results}

The emergency treatment service is analyzed in the following four steps. At first, it is emergency detection. The second step is a call for help to medical institutes and contacting a family member, and also a special healthcare vehicle service. The third step is transportation by the ambulance to the hospital. The last step is preparation of the rescue. The statistical results of the time spent in the whole process are shown in Table 6.

In this test, the distance between CVD patient and hospital was five kilometers and when systolic blood pressure is higher than the standard value by 10 points or diastolic blood pressure is lower than the standard value by 10 points the emergency situation will be triggered.

\begin{tabular}{|l|l|c|c|c|}
\hline Emergency Treatment & \multicolumn{2}{|c|}{ Traditional Rescue Service } & \multicolumn{2}{c|}{ EMS Based Rescue Service } \\
\hline Emergency Detection & Seek help & $\begin{array}{c}>=1 \\
\text { minutes }\end{array}$ & Sensors & $\begin{array}{c}<=30 \\
\text { Seconds }\end{array}$ \\
\hline \multirow{2}{*}{ Call for Help } & $\begin{array}{l}\text { 1. Call 120 first aid } \\
\text { 2. Contact family members } \\
\text { 3. Special vehicle service }\end{array}$ & $\begin{array}{c}>=1 \\
\text { minutes }\end{array}$ & Fog System & $\begin{array}{c}<=30 \\
\text { seconds } \\
<=60 \\
\text { seconds }\end{array}$ \\
\cline { 2 - 3 } Moving (5 km) & Round Trip Delay & $\begin{array}{c}>=30 \\
\text { minutes }\end{array}$ & $\begin{array}{c}\text { Mobile APP, } \\
\text { Nearly One Way Delay }\end{array}$ & $\begin{array}{c}<=18 \\
\text { minutes }\end{array}$ \\
\cline { 1 - 5 } Preparation of Rescue & Situation analysis & $\begin{array}{c}>=3 \\
\text { minutes }\end{array}$ & Fog System & Gear up \\
\hline Total & \multicolumn{2}{|r|}{ Nearly 35 minutes } & \multicolumn{2}{|c|}{ Hardly 19 minutes } \\
\hline
\end{tabular}

Table 6. Comparative analysis of rescue service time

\subsection{PHR and EHR Based Healthcare Services}

The structure of ontology based cohort DB was provided for the PHR and EHR services [34], and the smart sensor and mobile device based PHR general services and medical institutions providing EHR extended services are proposed, as shown in Figure 10. In this case the additional healthcare services can provide an effective emergency monitoring and diseases prediction services about a personal daily life for medical institutes.

\section{CONCLUSIONS}

The goal of this research is the cross-application of the advanced information and communication technology and medical technology to provide EMS and DPS, and use these services to reduce the sudden mortality rate.

The proposed EMDPS platform embodies three aspects of the technological advantages. The first one is the network service, the fog computing layer in the proposed network architecture can solve network latency problem, and the core layer provides advanced data security and delivery service, and the mobile edge computing layer provides real-time healthcare services at hand. The second one is the proposed 


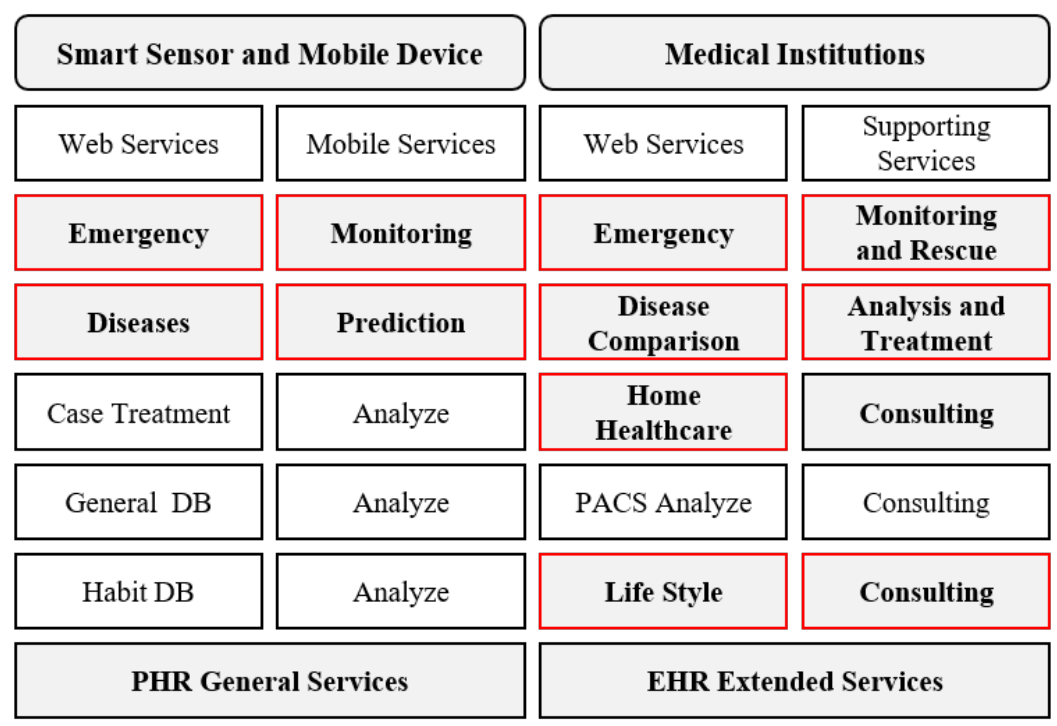

Figure 10. The PHR and EHR based healthcare services

EMDPS platform architecture, it is focused on the EM and DP services based on EMDPS integrated MAPE-K model. From the EM method, DP method, the plan management and the system management the platform has greatly advanced. The last one is mobile EMDP service model, it can provide real-time healthcare services ubiquitously.

Although the proposed EMDPS is a scientific platform advanced in specific services, still more detailed and deep research is needed to achieve the accurate disease prediction and prevention. Hence, the goal of the extended future research is to develop a biotechnology integrated healthcare platform to get closer to the precision medicine.

\section{Acknowledgements}

This paper was supported by the Scientific Research Foundation of Shandong Natural Science (No. ZR2013DMOII), Shandong Provincial Key R \& D (No. 2016GGX105013) and Shandong University of Science and Technology for Recruited Talents.

\section{REFERENCES}

[1] Ding, S. M.: Shandong Annual Conference on Diseases and Health Status of Residents. The State Council Information Office of the People's Republic of China. Available at: http://www.scio.gov.cn, 2017 (in Chinese). 
[2] KIM, D. H.-KIM, S. S.: PHR Related Recent Research Trend from the Smart Medical Platform Activity Point. Journal of Communication and Networks, Korea, Vol. 35, 2018, pp. 10-17.

[3] Kumari, A.-Tanwar, S.-Tyagi, S.-Kumar, N.: Fog Computing for Healthcare 4.0 Environment: Opportunities and Challenges. Computers and Electrical Engineering, Vol. 72, 2018, pp. 1-13, doi: 10.1016/j.compeleceng.2018.08.015.

[4] JeOng, Y.S.: An Efficient IoT Healthcare Service Management Model of Location Tracking Sensor. Journal of Digital Convergence, Vol. 14, 2016, No. 3, pp. 261-267, doi: 10.14400/JDC.2016.14.3.261 (in Korean).

[5] Jeong, Y.S.: Subnet Generation Scheme Based on Deep Learing for Healthcare Information Gathering. Journal of Digital Convergence, Vol. 15, 2017, No. 3, pp. 221-228, doi: 10.14400/JDC.2017.15.3.221 (in Korean).

[6] Lopez-Barbosa, N.-Gamarra, J. D.-Osma, J. F.: The Future Point-of-Care Detection of Disease and Its Data Capture and Handling. Analytical and Bioanalytical Chemistry, Vol. 408, 2016, pp. 2827-2837, doi: 10.1007/s00216-015-9249-2.

[7] Kumar, R.-Ganapathy, G.-Kang, J.: A Novel Architecture for Mobile Crowd and Cloud Computing for Health Care. International Journal of Advanced Culture Technology, Vol. 6, 2018, No. 4, pp. 226-232, doi: 10.17703//IJACT2018.6.4.226.

[8] Xu, B. Y.-Xu, L. D.-CAI, H. M.-Jiang, L. H.: Architecture of M-Health Monitoring System Based on Cloud Computing for Elderly Homes Application. Second International Conference on Enterprise Systems, Shanghai, China, 2014, pp. 45-50, doi: $10.1109 / \mathrm{ES} .2014 .11$.

[9] Sun, J.-WAng, X. J.-Wang, S. P.-Ren, L. L.: A Searchable Personal Health Records Framework with Fine-Grained Access Control in Cloud-Fog Computing. PLoS One, Vol. 13, 2018, No. 11, Art. No. e0207543, doi: 10.1371/journal.pone.0207543

[10] He, X.Z.-Zhao, L.: A Data Management and Analysis System in Healthcare Cloud. 2013 International Conference on Service Sciences (ICSS), 2013, pp. 164-169, doi: $10.1109 /$ ICSS.2013.27.

[11] Sun, Y.Y.-WAng, Y.H.-Li, M. M.-Cheng, K. L.-ZhaO, X. Y.Zheng, Y.-Liu, Y.-LeI, S. Y.-WAng, L.: Long-Term Trends of Liver Cancer Mortality by Gender in Urban and Rural Areas in China: An Age-Period-Cohort Analysis. BMJ Open, Vol. 8, 2018, Art. No. e020490, 8 pp., doi: 10.1136/bmjopen2017-020490

[12] Åström, F.-Koker, R.: A Parallel Neural Network Approach to Prediction of Parkinson's Disease. Expert Systems with Applications, Vol. 38, 2011, No. 10, pp. 12470-12474, doi: 10.1016/j.eswa.2011.04.028.

[13] Sargent, D. J.: Comparison of Artificial Neural Networks with Other Statistical Approaches: Results from Medical Data Sets. Cancer, Vol. 91, 2001, No. 8 Suppl., pp. 1636-1642.

[14] Perai, A. H.-Nassiri Moghaddam, H.-Asadpour, S.-Bahrampour, J.Mansoori, G.: A Comparison of Artificial Neural Networks with Other Statistical Approaches for the Prediction of True Metabolizable Energy of Meat and Bone Meal. Poultry Science, Vol. 89, 2010, No. 7, pp. 1562-1568, doi: 10.3382/ps.2010-00639. 
[15] Azimi, I.-Anzanpour, A.-Rahmani, A. M.-Pahikkala, T.-Levorato, M.-Liljeberg, P.-Dutt, N.: HiCH: Hierarchical Fog-Assisted Computing Architecture for Healthcare IoT. ACM Transactions on Embedded Computing Systems, Vol. 16, 2017, No. 5s, Art. No. 174, 20 pp., doi: 10.1145/3126501.

[16] Nandyala, C.S.-Kim, H. K.: From Cloud to Fog and IoT-Based Real-Time U-Healthcare Monitoring for Smart Homes and Hospitals. International Journal of Smart Home, Vol. 10, 2016, No. 2, pp. 187-196, doi: 10.14257/ijsh.2016.10.2.18.

[17] Trung, T. Q.-LeE, N.E.: Flexible and Stretchable Physical Sensor Integrated Platforms for Wearable Human-Activity Monitoring and Personal Healthcare. Advanced Materials, Vol. 28, 2016, No. 22, pp. 4338-4372, doi: 10.1002/adma.201504244

[18] Wan, J.-Al-awlaki, M. A. A. H.-Li, M. S.-O'Grady, M.-Gu, X.Wang, J.-CaO, N.: Wearable IoT Enabled Real-Time Health Monitoring System. EURASIP Journal on Wireless Communications and Networking, Vol. 1, 2018, Art. No. 298, 10 pp., doi: 10.1186/s13638-018-1308-x.

[19] Woo, M. W.-Lee, J. W.-Park, K. H.: A Reliable IoT System for Personal Healthcare Devices. Future Generation Computer Systems, Vol. 78, 2018, No. 2, pp. 626-640, doi: 10.1016/j.future.2017.04.004

[20] Islam, M. S.-Islam, M. T.-Almutairi, A. F.-Beng, G. K.-Misran, N.Amin, N.: Monitoring of the Human Body Signal Through the Internet of Things (IoT) Based LoRa Wireless Network System. Applied Sciences, Vol. 9, 2019, No. 9, pp. 1884-1901, doi: 10.3390/app9091884.

[21] Kharel, J.-Reda, H.T.-Shin, S. Y.: Fog Computing-Based Smart Health Monitoring System Deploying LoRa Wireless Communication. IETE Technical Review, Vol. 36, 2019, No. 1, pp. 69-82, 2019, doi: 10.1080/02564602.2017.1406828.

[22] Samarah, S.-Al Zamil, M. G. A.-Rawashdeh, M.-Hossain, M. S.-Muhammad, G.-Alamri, A.: Transferring Activity Recognition Models in FOG Computing Architecture. Journal of Parallel and Distributed Computing, Vol. 122, 2018, pp. 122-130, doi: $10.1016 /$ j.jpdc.2018.07.020

[23] Wen, L. R.-Yang, S. M.-Lee, B. M.: Cloud Platform Based Mobile Service for Aging Gerneration Healthcare Management. International Journal of Multimedia and Ubiquitous Engineering, Vol. 11, 2016, No. 11, pp. 235-246.

[24] Ganatra, N. P.-Patel, R. S.: Proposed Customized Architecture of Mobile Cloud Computing in Health Care Domain. International Journal of Advanced Research in Computer Science, Vol. 8, 2017, No. 5, pp. 876-879.

[25] Zhang, W.-Lin, Y.P.-Wu, J.-Zhou, T.: Inference Attack-Resistant E-Healthcare Cloud System with Fine-Grained Access Control. IEEE Transactions on Services Computing (Early Access), 2018, pp. 1-14, doi: 10.1109/TSC.2018.2790943.

[26] Isa, I. S. M.-Musa, M. O. I.-El-Gorashi, T. E. H.-Lawey, A. Q.-ElmirGHANI, J. M. H.: Energy Efficiency of Fog Computing Health Monitoring Applications. $201820^{\text {th }}$ International Conference on Transparent Optical Networks (ICTON), Bucharest, Romania, 2018, doi: 10.1109/ICTON.2018.8473698.

[27] Cao, G.-Liu, J.: An IoT Application: Health Care System with Android Devices. In: Gervasi, O. et al. (Eds.): Computational Science and Its Applications - 
ICCSA 2016. Springer, Cham, Lecture Notes in Computer Science, Vol. 9786, 2016, pp. 563-571, doi: 10.1007/978-3-319-42085-1_46.

[28] Zamanifar, A.-Nazemi, E.-Vahidi-Asl, M.: DMP-IOT: A Distributed Movement Prediction Scheme for IoT Health-Care Applications. Computers and Electrical Engineering, Vol. 58, 2017, pp. 310-326, doi: 10.1016/j.compeleceng.2016.09.015.

[29] Vizcarrondo, J.-Aguilar, J.-Exposito, E.-Subias, A.: MAPE-K as a Service-Oriented Architecture. IEEE Latin America Transactions, Vol. 15, 2017, No. 6, pp. 1163-1175, doi: 10.1109/TLA.2017.7932705

[30] IBM Corporation: An Architectural Blueprint for Autonomic Computing. White Paper, Third Edition, 2005.

[31] Bonomi, F.-Milito, R.-Natarajan, P.-Zhu, J.: Fog Computing: A Platform for Internet of Things and Analytics. In: Bessis, N., Dobre, C. (Eds.): Big Data and Internet of Things: A Roadmap for Smart Environments. Springer, Cham, Studies in Computational Intelligence, Vol. 546, 2016, pp. 169-186, doi: $10.1007 / 978-$ 3-319-05029-4_7.

[32] Chernyavskiy, P.-Little, M. P.-Rosenberg, P. S.: Correlated Poisson Models for Age-Period-Cohort Analysis. Statics in Medicine, Vol. 37, 2017, No. 3, pp. 405-424, doi: 10.1002/sim.7519.

[33] Fu, W. J.: Ridge Estimator in Singular Design with Application to Age-PeriodCohort Analysis of Disease Rates. Communications in Statistics - Theory and Methods, Vol. 29, 2000, No. 2, pp. 263-278, doi: 10.1080/03610920008832483.

[34] Wen, L. R.-Yang, S. M.-Lee, B. M.: Healthcare Platform and Big Data Analysis Based Personal Fitness Healthcare Service Model. International Journal of BioScience and Bio-Technology, Vol. 8, 2016, No. 5, pp. 115-128, doi: 10.14257/ijbsbt.2016.8.5.11. 

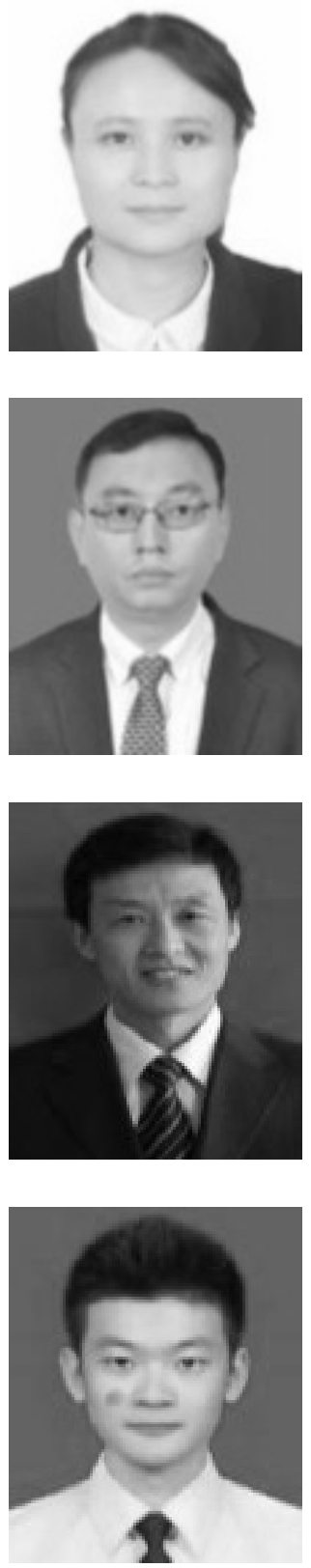

Zhancui LI received her B.Sc. degree from the Military Medical University, Beijing, China, in 2003. She is currently working as a senior technologist at the Department of Magnetic Resonance Surgery, the 960 Hospital of Joint Logistics Support Force of PLA, Shandong Taian, China. Her main research includes medical image processing and medical imaging diagnosis.

Longri WEN received his Ph.D. degree in computer science from the Soongsil University, South Korea, in 2017. He is currently working as Assistant Professor at the Shandong University of Science and Technology, Taian Shandong, China. His main research includes precision medicine, ICT and medical technology and bio technology integrated application.

Jimin LIU received his Ph.D. degree in geodesy and surveying engineering from the Shandong University of Science and Technology. He is currently working as the Director of the Information Engineering Department and Professor at the Shandong University of Science and Technology. His main research includes data mining and machine learning algorithms.

Quanqiu JIA received his B.Sc. degree in computer science from the Shandong University of Science and Technology. He is currently pursuing his M.Sc. degree at Shandong University of Science and Technology. His main research includes medical data mining and big data analysis. 


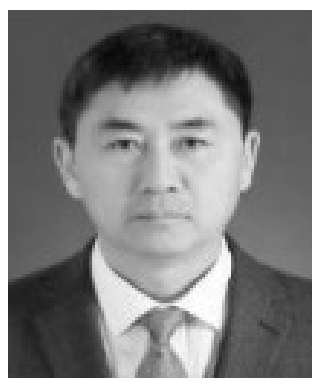

Chengri ChE received his M.Dr. degree in medical management from the Chungnam National University, South Korea in 2002. He is currently working as the Director of thoracic surgery and Professor at the Yanbian University Affiliated Hospital. He is the first batch of outstanding young and middle-aged talents of the Jilin province in China.

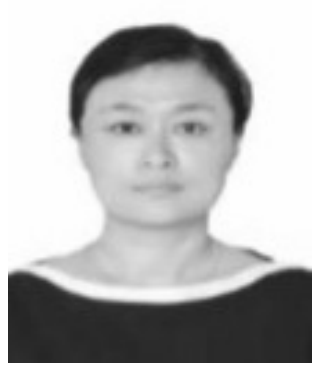

Chengfeng SHI received her B.Sc. degree from the Taishan Medical University, Shandong Taian, China, in 2010. She is currently working at the Department of Maternal Healthcare, Maternal and Child Health Hospital, Shandong Taian, China. Her main research includes personal healthcare and disease prevention technology.

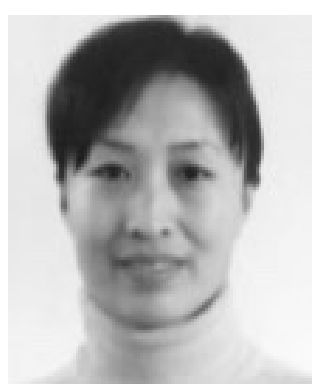

Haiying CAI received her B.Sc. degree from the Jining Medical University, Jining Shandong, China, in 2008. She is currently working at the Department of Cancer Prevention and Treatment Institute, Shandong Taian, China. Her main research includes cancer disease prediction and disease prevention technology. 